\title{
Effect of targeted fluid minimization and diuretic therapy on hospital- acquired heart failure and adverse outcomes in patients with no preexisting cardiac dysfunction
}

Kwannate Intarawongchot

Chulalongkorn University

Sutep Gonlachanvit

Chulalongkorn University

Sarinya Puwanant ( $\sim$ spuwanant@gmail.com )

Chulalongkorn University

\section{Research Article}

Keywords: Intravenous fluid, fluid balance, acute heart failure, outcomes

Posted Date: June 9th, 2021

DOI: https://doi.org/10.21203/rs.3.rs-584690/v1

License: (a) (1) This work is licensed under a Creative Commons Attribution 4.0 International License.

Read Full License 


\section{Abstract}

Background This study aimed to investigate: 1) the incidence of hospital-acquired heart failure (HF) in patients with no preexisting cardiac disorder, and 2) whether the use of an intervention protocol comprised of targeted fluid minimization and diuretic therapy can reduce the incidence of hospitalacquired HF and adverse outcomes.

Methods We conducted a single-center, open-labeled, prospective cohort study enrolling patients with no preexisting cardiac dysfunction who were admitted to the medical wards and had a positive intravenous fluid balance $>4 L$ within 3 days. We assigned patients in a 1:1 ratio to the intervention protocol (intervention group) or usual care. The primary outcome was hospital-acquired HF. The secondary outcomes included in-hospital mortality, intensive critical unit (ICU) admission, mechanical ventilator usage, or prolonged hospital stay $>30$ days.

Results A total of 98 patients (mean age $66 ; 52 \%$ male) were enrolled (intervention group, 49; usual care group, 49). The incidence of hospital-acquired HF among all patients was $21 \%$. Patients with hospitalacquired had higher rates of in-hospital mortality ( $48 \%$ vs. $13 \%$; $p=0.001)$, ICU admission ( $33 \%$ vs. $10 \%$; $p$ $=0.010)$ and mechanical ventilator usage $(62 \%$ vs. $35 \% ; p=0.027)$. Prolonged hospital stay $>30$ days rates were similar in patients with and without hospital-acquired HF. Hospital-acquired HF was not found statistically different between groups (intervention group $18 \%$ vs. usual care group $25 \% ; p=0.460$ ). Patients in the intervention group did have lower rates of subsequent ICU admission ( $8 \%$ vs.23\%; $p=$ $0.049)$ and hospitalizattion $>30$ days. ( $8 \% v s .27 \% ; p=0.018)$ compared with the usual care group. Inhospital mortality and mechanical ventilator usage were not different between groups.

Conclusions The incidence of hospital-acquired HF in patients with no preexisting cardiac dysfunction who had a positive cumulative fluid balance of $>4 \mathrm{~L}$ within 3 days was not uncommon, about one in five patients. Hospital-acquired HF can lead to increased in-hospital mortality and co-morbidities. Targeted fluid minimization and diuretics did show a reduced rate of ICU admission and prolonged hospitalization. However, no statistical difference in rates of hospital-acquired HF and in-hospital mortality compared to the control group were found.

\section{Introduction}

Acute de novo heart failure (HF) developing after hospital admission or hospital-acquired heart failure (HF) is associated with a greater length of hospital stay and mortality. ${ }^{1-6}$ The mortality of patients with hospital-acquired HF has been shown to be approximately 3 times the rate preadmission HF or general acute decompensated HF. ${ }^{4}$ Previous studies have shown that inappropriate intravenous (IV) fluid and failure to restart regular diuretics were related to hospital-acquired HF. ${ }^{5}$ This study aimed to investigate This study aimed to investigate: 1) the incidence of hospital-acquired HF in patients with no preexisting cardiac disorder, and 2) whether the use of an intervention protocol comprised of targeted fluid 
minimization and diuretic therapy can reduce the incidence of hospital-acquired HF and adverse outcomes.

\section{Methods}

\section{Study participants}

We conducted a single-center, open-labeled, prospective cohort study of adult patients aged 18 years or more with no preexisting cardiac diseases who were admitted to the medical wards and had a positive IV fluid balance of greater than 4 liters $(L)$ in 3 days. We excluded patients with a history of heart failure, ischemic heart disease, atrial fibrillation, ventricular arrhythmia, valvular heart disease, cardiomyopathy, congenital heart diseases, pericardial disease, hyperthyroidism, shock, pancreatitis, and patients with chronic kidney disease stage 4-5. We assigned patients in a 1:1 ratio to either use the protocol (intervention group) or usual care each for 3 days. All participants provided written informed consents. The study protocol was approved by the Institutional Review Board, Faculty of Medicine, Chulalongkorn University, Bangkok, Thailand. (IRB No. 710/61). All research was performed in accordance with relevant guidelines and regulations.

\section{Intervention Protocol}

The protocol was activated to the in-patient physician in the intervention group when a positive cumulative fluid balance of greater than $4 \mathrm{~L}$ in 3 days during hospitalization. The protocol was comprised of: (1) reduction of IV fluid administration by at least $30 \%$ of the total fluid volume on 1 day before the protocol activation; and/or (2) administering IV diuretic therapy to achieve a zero or negative net fluid balance.

\section{Outcomes}

The primary outcome was hospital-acquired HF which was defined as acute de novo HF developing after hospital admission. Acute HF was defined by the Framingham criteria. ${ }^{7}$ Secondary outcomes included in-hospital mortality, intensive critical unit (ICU) admission, mechanical ventilator usage, and prolonged hospitalization $>30$ days.

\section{Availability of data}

Data is available upon request.

\section{Statistical analysis}

Descriptive data were presented by frequency, percentage, median and range, and mean \pm standard deviation (SD) depending upon data distribution. Differences in continuous variables were determined by a student's t-test or a Wilcoxon-rank-sum test. Categorical variables were compared using a Chi-square test or a Fisher's exact test. To identify the predictors of hospital-acquired HF and other adverse hospital outcomes, univariate analysis was performed comparing hospital-acquired HF occurrence with demographic, clinical, and echocardiographic characteristics. A p-value of $<0.05$ was considered 
statistically significant. Statistical analyses were conducted using the SPSS statistical analysis package (version 18.0.0; SPSS Inc., Chicago, Illinois, USA).

\section{Results}

\section{Patient Characteristics}

A total of 98 patients (mean age $66 ; 52 \%$ male) were enrolled with 49 patients in both the intervention and usual care groups. The most common principal diagnosis for hospitalization was hepatobiliary tract and gastrointestinal disorders followed by respiratory tract infection or pneumonia. Table 1 shows baseline characteristics in overall patients and patients in each assigned group. Average age showed no differences between groups. Differences were found between groups with intervention group patients having higher rates in four characteristics: 1$)$ male $(69 \%$ vs. $35 \% ; p=0.01), 2)$ greater body surface area (BSA) (1.57 vs. $1.48 ; p=0.31)$, and 3 ) body weight $(\mathrm{kg})(55 \mathrm{vs.} 50 ; \mathrm{p}=0.49)$. Other demographic characteristics and comorbidities were similar in both groups. 
Table 1

Baseline Characteristics

\begin{tabular}{|c|c|c|c|c|}
\hline Factors & $\begin{array}{l}\text { Total } \\
(\mathrm{N}=98)\end{array}$ & $\begin{array}{l}\text { Intervention } \\
(\mathrm{N}=49)\end{array}$ & $\begin{array}{l}\text { Usual Care } \\
(\mathrm{N}=49)\end{array}$ & P-value \\
\hline Age (years) & $66 \pm 18$ & $64 \pm 18$ & $67 \pm 17$ & 0.332 \\
\hline$<60$ years & $29(30 \%)$ & $17(35 \%)$ & $12(25 \%)$ & 0.269 \\
\hline $60-70$ years & $25(26 \%)$ & $11(23 \%)$ & $14(29 \%)$ & 0.487 \\
\hline$>70$ years & $44(45 \%)$ & $21(43 \%)$ & $23(47 \%)$ & 0.685 \\
\hline Male $(n, \%)$ & $51(52 \%)$ & $34(69 \%)$ & $17(35 \%)$ & $0.001 *$ \\
\hline Weight (kg) & $53 \pm 12$ & $55 \pm 12$ & $50 \pm 11$ & $0.049 *$ \\
\hline Body surface area $\left(\mathrm{m}^{2}\right)$ & $1.52 \pm 0.19$ & $1.57 \pm 0.19$ & $1.48 \pm 0.19$ & $0.031^{*}$ \\
\hline Body mass index $\left(\mathrm{kg} / \mathrm{m}^{2}\right)$ & $20 \pm 4$ & $21 \pm 4$ & $20 \pm 4$ & 0.276 \\
\hline Active smoker (n, \%) & $11(11 \%)$ & $3(6 \%)$ & $8(16 \%)$ & 0.109 \\
\hline \multicolumn{5}{|l|}{ Comorbidities (n, \%) } \\
\hline Diabetes & $18(18 \%)$ & $10(20 \%)$ & $8(16 \%)$ & 0.602 \\
\hline Hypertension & $28(29 \%)$ & $16(33 \%)$ & $12(25 \%)$ & 0.371 \\
\hline Anemia & $75(77 \%)$ & $37(76 \%)$ & $38(78 \%)$ & 0.812 \\
\hline Cirrhosis & $9(9 \%)$ & $7(14 \%)$ & $2(4 \%)$ & 0.080 \\
\hline Cancer & $24(25 \%)$ & $8(16 \%)$ & $16(33 \%)$ & 0.060 \\
\hline \multicolumn{5}{|l|}{ Principle Admission Diagnosis } \\
\hline $\begin{array}{l}\text { Hepatobiliary tract disease and } \\
\text { Gastrointestinal disorders }\end{array}$ & $25(26 \%)$ & $9(18 \%)$ & $16(33 \%)$ & 0.105 \\
\hline Respiratory tract infection & $24(24 \%)$ & $10(20 \%)$ & $14(29 \%)$ & 0.375 \\
\hline Infection & $21(21 \%)$ & $13(27 \%)$ & $(16 \%)$ & 0.218 \\
\hline Cancer-related problems & $9(9 \%)$ & $4(8 \%)$ & $5(10 \%)$ & 0.726 \\
\hline Central nervous system disorders & $5(5 \%)$ & $1(2 \%)$ & $4(8 \%)$ & 0.168 \\
\hline Pulmonary disorders & $5(5 \%)$ & $1(2 \%)$ & $4(8 \%)$ & 0.168 \\
\hline Metabolic disorders & $5(5 \%)$ & $1(2 \%)$ & $4(8 \%)$ & 0.168 \\
\hline * $p$ value $<0.05$ & & & & \\
\hline
\end{tabular}




\begin{tabular}{|lllll|}
\hline Factors & $\begin{array}{l}\text { Total } \\
(\mathbf{N}=98)\end{array}$ & $\begin{array}{l}\text { Intervention } \\
(\mathbf{N}=49)\end{array}$ & $\begin{array}{l}\text { Usual Care } \\
(\mathbf{N}=49)\end{array}$ & P-value \\
\hline $\begin{array}{l}\text { Rheumatologic and } \\
\text { musculoskeletal disorders }\end{array}$ & $4(4 \%)$ & $1(2 \%)$ & $3(6 \%)$ & 0.307 \\
\hline Hemoglobin $(\mathrm{g} / \mathrm{dL})$ & $10.28 \pm 2.47$ & $10.22 \pm 2.80$ & $10.33 \pm 2.12$ & 0.830 \\
\hline Creatinine $(\mathrm{mg} / \mathrm{dL})$ & $1.04 \pm 0.67$ & $1.07 \pm 0.76$ & $1.01 \pm 0.57$ & 0.654 \\
\hline Albumin $(\mathrm{g} / \mathrm{dL})$ & $3.04 \pm 0.65$ & $3.14 \pm 0.63$ & $2.95 \pm 0.66$ & 0.150 \\
\hline Positive fluid balance $(\mathrm{ml})$ & $4303 \pm 733$ & $4233 \pm 472$ & $4375 \pm 924$ & 0.340 \\
\hline * $\mathrm{p}$ value $<0.05$ & & & & \\
\hline
\end{tabular}

\section{Protocol Achievement}

The mean of positive IV fluid balance (liters) was 4.30, 4.23, and 4.38 among all patients, intervention group, and usual care group, respectively. Of 49 patients in the intervention group, reduction in IV fluid administration of at least $30 \%$ of fluid volume on 1 day before the protocol activation was commenced in 47 patients (98\%), and IV diuretics, mean dose of $60 \mathrm{mg}$ of furosemide, was administered in 40 patients $(85 \%)$. All patients in the intervention group achieved a zero or negative fluid balance within 48 hours after the protocol activation.

\section{Outcomes}

Table 2 shows hospital outcomes in overall patients and patients in each assigned group. Hospitalacquired HF, HF-related mortality, in-hospital mortality, and mechanical-ventilation rates were similar in both groups. Patients in the intervention group did show lower rates of subsequent ICU admission ( $8 \%$ vs. $23 \% ; p=0.049)$ and hospital stay $>30$ days. $(8 \%$ vs. $27 \% ; p=0.018)$ than those in the usual care group. 
Table 2

Outcomes between patients in the intervention and the usual care groups.

\begin{tabular}{|lllll|}
\hline Outcomes & $\begin{array}{l}\text { Total } \\
\mathbf{N = 9 8 )}\end{array}$ & $\begin{array}{l}\text { Intervention } \\
\mathbf{( N = 4 9 )}\end{array}$ & $\begin{array}{l}\text { Usual Care } \\
\mathbf{( N = 4 9 )}\end{array}$ & P-value \\
\hline Hospital-acquired heart failure & $21(21 \%)$ & $9(18 \%)$ & $12(25 \%)$ & 0.4600 \\
\hline Heart failure-related mortality $(\mathrm{n}, \%)$ & $3(3 \%)$ & $1(2 \%)$ & $2(4 \%)$ & 0.5570 \\
\hline In-hospital mortality $(\mathrm{n}, \%)$ & $20(21 \%)$ & $8(16 \%)$ & $12(25 \%)$ & 0.3161 \\
\hline Intensive critical unit admission $(\mathrm{n}, \%)$ & $15(15 \%)$ & $4(8 \%)$ & $11(23 \%)$ & $0.0495^{*}$ \\
\hline Mechanical-ventilator requirement & $40(41 \%)$ & $16(33 \%)$ & $24(49 \%)$ & 0.1000 \\
\hline Length of stay > 30 days (n, \%) & $17(18 \%)$ & $4(8 \%)$ & $13(27 \%)$ & $0.0184^{\star}$ \\
\hline Length of stay (days) & $17(2-90)$ & $12(2-40)$ & $23(2-90)$ & $0.0005^{\star}$ \\
\hline * $p$ value $<0.05$ & & & & \\
\hline
\end{tabular}

\section{Hospital-acquired HF}

Hospital-acquired HF occurred in $21 \%$ of the study patients. Among the 21 patients with hospital-acquired HF, left ventricular ejection fraction (LVEF) was evaluated in 16 patients (76\%). Mean LVEF was $64 \pm 6 \%$. Hypertensive heart disease was evident in 10 patients (63\%). Patients with hospital-acquired HF had higher rates of in-hospital mortality ( $48 \%$ vs. $13 \%$; $p=0.001)$, ICU admission $(33 \%$ vs. $10 \% ; p=0.010)$ and use of mechanical ventilator $(62 \%$ vs. $35 \% ; p=0.027)$. Length of hospital stay $>30$ days rates were similar in patients with and without hospital-acquired HF. (Table 3 ) Results from the univariate analysis show no specific patient baseline characteristics or IV cumulative fluid volume associated with hospitalacquired HF. 
Table 3

Outcomes between patients with and with no hospital-acquired HF

\begin{tabular}{|c|c|c|c|}
\hline Outcomes & $\begin{array}{l}\text { Hospital-acquired HF ( } \\
=21) \\
(\mathrm{N}=49)\end{array}$ & $\begin{array}{l}\text { No hospital-acquired HF ( } N \\
=77)\end{array}$ & P-value \\
\hline $\begin{array}{l}\text { Heart failure related mortality } \\
(n, \%)\end{array}$ & $3(14 \%)$ & 0 & $0.0019 *$ \\
\hline In-hospital mortality (n, \%) & $10(48 \%)$ & $10(13 \%)$ & $0.0005^{\star}$ \\
\hline $\begin{array}{l}\text { Intensive critical unit admission } \\
(\mathrm{n}, \%)\end{array}$ & $7(33 \%)$ & $8(10 \%)$ & $0.0096^{*}$ \\
\hline $\begin{array}{l}\text { Mechanical-ventilator } \\
\text { requirement }\end{array}$ & $13(62 \%)$ & 27 (35\%) & $0.0265^{\star}$ \\
\hline Length of stay $>30$ days $(n, \%)$ & $4(19 \%)$ & $13(17 \%)$ & 0.8164 \\
\hline * $p$ value $<0.05$ & & & \\
\hline
\end{tabular}

\section{Discussion}

The major findings of our study are: (1) the incidence of hospital-acquired HF was $21 \%$ in adult hospitalized patients with no previous history of cardiac dysfunction who had a positive cumulative IV fluid balanced of $>4 \mathrm{~L}$ in 3 days; (2) most of the hospital-acquired HF patients had HF with preserved LVEF and reported high in-hospital mortality and low HF-related mortality; and (3) targeted fluid minimization and IV furosemide at the mean dose of $60 \mathrm{mg}$ did not reduce either hospital-acquired HF rates, in-hospital mortality, or the need for mechanical ventilators. Patients managed by the intervention protocol did have a shorter length of stay and less frequent ICU admission rates.

\section{Hospital-acquired HF}

Data on incidence of hospital-acquired HF among medical hospitalized patients with no preexisting cardiac disorders is currently limited. Previous study reported a $1 \%$ incidence of hospital-acquired HF in medical, oncology, surgical, and orthopedic wards. ${ }^{5}$ The median IV fluid volume administered 1 day and 2 days before the onset of HF was 2758 and $2000 \mathrm{~mL}$, respectively. The authors demonstrated that administration of $>2 \mathrm{~L}$ of IV fluid on the day before the onset of HF was associated with hospitalacquired HF (OR 3.5, 95\% Cl 1.2-9.8). ${ }^{5}$ The $21 \%$ incidence of hospital-acquired HF in our cohort was higher than the Plant et al study. ${ }^{5}$ In our study, about $70 \%$ of patients aged over 60 years old and had anemia. Recent studies have shown that anemia is common in patients with HF and is associated with worsening natural course of HF, increased HF admission, and increased mortalities. ${ }^{8,9}$ These factors may influence the divergence of outcomes between studies. Patel et al. reported that $7 \%$ of acute HF 
hospitalizations in patients with the age over 55 had post-admission onset of acute HF or hospitalacquired HF. ${ }^{4}$ However, they did not examine the association between fluid volume and hospital-acquired HF. Branan et al. studied the hidden fluid administration, including IV medication, line flushes, blood products, and enteral nutrition, and the development of fluid overload, defined as a positive accumulative fluid balance producing weight gain over $10 \%$ from baseline, in 219 ICU patients. ${ }^{2}$ They found that the volume of hidden fluid was associated with fluid overload. The incidence of fluid overload in the high hidden fluid volume group ( $>4.4 \mathrm{~L}$ ) was $25 \%$ which was comparable to the HF rate in our study. The study did not report the rate of HF among those with fluid overload which would have allowed for better comparison. Pathophysiology involving hospital-acquired HF may include: 1) tachycardia or afterload mismatch in preexisting diastolic dysfunction or hypertensive heart disease, 2) myocardial ischemia due to an imbalance between the coronary blood supply and demand in hospitalized conditions, or 3) stressinduced cardiomyopathy.

We found that hospital-acquired HF in our patients was associated with adverse outcomes. Previous studies have shown that hospital-acquired HF carried high morbidity and in-hospital mortality. ${ }^{1,2,4-6}$ Patel et al. demonstrated that short-term mortality in hospital-acquired HF was 3 times the rate of preadmission HF. ${ }^{4}$ Patients with hospital-acquired HF had a worse short-term prognosis compared with those with preadmission HF. The authors reported in-hospital mortality of $16 \%$. Similarly, Plant et al found that length of stay and mortality in patients with hospital-acquired HF were significantly higher than those in the age-gender matched control group. ${ }^{5}$ They reported a mortality rate of $13 \%$ in the hospitalacquired HF group. Taylor et al. also found that patients with hospital-acquired HF significantly had a higher death rate (26\%) compared with the community group (6\%). ${ }^{6}$ In this study, the mortality in the hospital-acquired HF group was $48 \%$. This is significantly higher than those reported by other studies. The possible explanation for the high mortality in our study may include a higher proportion of elderly patients with cancer, pneumonia, and pulmonary disease, a higher prevalence of concomitant anemia, and a higher rate of mechanical ventilator usage. These higher rates of subsequent ICU transfer and mechanical ventilator usage is in concordance with results from previous studies. 4,5

\section{Intervention Protocol}

Previous studies have shown that IV fluid volume is associated with hospital-acquired HF, fluid overload, and adverse outcomes ${ }^{1-6}$. Plant et al. found that failure to restart regular diuresis was common in the hospital-acquired HF group. ${ }^{5}$ We developed an intervention protocol comprised of minimizing fluid administration and administering of IV diuretics to patients who were at risk of fluid overload and HF. We found that the use of this protocol in hospitalized patients with no pre-existing cardiovascular diseases who received a positive cumulative fluid balance greater than $4 \mathrm{~L}$ for 3 days was not associated with lower occurrence of hospital-acquired HF or reduced mortality compared to our control group who received standard care. Delivery of the intervention protocol was associated with less frequent ICU admission and shorter length of hospital stay. The possible reasons for the neutral effect of the protocol on hospital-acquired HF and in-hospital mortality may include: (1) the causes of death mostly did not relate to hospital-acquired HF, but rather to other underlying disease and comorbidities; (2) adherence to 
IV diuretics reached $85 \%$ of the interventional group; (3) volume of fluid minimization and diuretic dose in the protocol may be suboptimal; and (4) afterload optimization was not included and examined in the present study. Notably, a higher positive fluid balance or a need for large volume fluid in medical patients may be an indirect marker of more critically ill patients.

\section{Study limitations}

First, we did not investigate the natriuretic peptide or left ventricular ejection fraction in all patients.

Second, we could not identify and exclude patients that may have concealed cardiac dysfunction. Third, the proportion of male, body weight, and BSA were different between groups. Lastly, this study is limited to patients with no preexisting cardiac conditions who were hospitalized in medical wards. Further study with a larger study population and improvement of the intervention protocol is required.

\section{Conclusion}

We demonstrated that the incidence of hospital-acquired HF in medical patients with no preexisting cardiac dysfunction who had a positive cumulative IV fluid balance of greater than $4 \mathrm{~L}$ in 3 days after hospitalization was not uncommon, about one in five patients. Hospital-acquired HF can lead to increased in-hospital mortality and comorbidities, including a need for mechanical ventilator and ICU admission. The protocol comprised of targeted fluid minimization and diuretic therapy did not affect hospital-acquired HF and adverse outcomes.

\section{Abbreviations}

BSA Body surface area

HF Heart failure

IV Intravenous

ICU Intensive care unit

IG Intervention group

USC Usual care group

\section{Declarations}

ACKNOWLEDGEMENT: Authors would like to thank the research team of the Department of Medicine, Faculty of Medicine, Chulalongkorn University for editing the final manuscript.

FUNDING: This research was supported by the Ratchadapisek Sompoch Endowment Fund (2018), Chulalongkorn University [Grant No. RA 62/107]. 
CONTRIBUTORSHIP: Conceptualization of the work: KI, SG, and SP. Conduct of the work and data acquisition: KI and SP. Data analysis and interpretation: KI and SP. Drafting the original work: $\mathrm{KI}$ and SP. Critical reviewing the work and providing input: KI, SG, and SP. Final approval: KI, SG, and SP.

\section{References}

1. Bikdeli B, Strait KM, Dharmarajan K, et al. Intravenous fluids in acute decompensated heart failure. JACC Heart Fail. 2015;3(2):127-133.

2. Branan T, Smith SE, Newsome AS, Phan R, Hawkins WA. Association of hidden fluid administration with development of fluid overload reveals opportunities for targeted fluid minimization. SAGE Open Med. 2020;8:2050312120979464.

3. Khan RA, Khan NA, Bauer SR, et al. Association Between Volume of Fluid Resuscitation and Intubation in High-Risk Patients With Sepsis, Heart Failure, End-Stage Renal Disease, and Cirrhosis. Chest. 2020;157(2):286-292.

4. Patel MD, Kalbaugh CA, Chang PP, et al. Characteristics and outcomes of patients with acute decompensated heart failure developing after hospital admission. Am J Cardiol. 2014;114(10):15301536.

5. Plant LD, Taylor DM, Worland T, et al. Development of Acute Decompensated Heart Failure Among Hospital Inpatients: Incidence, Causes and Outcomes. Heart Lung Circ. 2019;28(3):406-413.

6. Taylor DM, Fui MN, Chung AR, Gani L, Zajac JD, Burrell LM. A comparison of precipitants and mortality when acute decompensated heart failure occurs in the community and hospital settings. Heart Lung Circ. 2012;21(8):439-443.

7. McKee PA, Castelli WP, McNamara PM, Kannel WB. The natural history of congestive heart failure: the Framingham study. N Engl J Med. 1971;285(26):1441-1446.

8. Ebner N, Jankowska EA, Ponikowski P, et al. The impact of iron deficiency and anaemia on exercise capacity and outcomes in patients with chronic heart failure. Results from the Studies Investigating Co-morbidities Aggravating Heart Failure. Int J Cardiol. 2016;205:6-12.

9. Klip IT, Jankowska EA, Enjuanes $C$, et al. The additive burden of iron deficiency in the cardiorenalanaemia axis: scope of a problem and its consequences. Eur J Heart Fail. 2014;16(6):655-662. 